\title{
Effects of Magnesium Modification on the Catalytic Performances of HZSM-5 Zeolite for the Conversion of Ethene to Propene
}

\author{
XU Lulu, ZHAO Zhenchao, ZHAO Rongrong, YU Rui, ZHANG Weiping * \\ State Key Laboratory of Fine Chemicals, School of Chemical Engineering, Dalian University of Technology, Dalian 116024, \\ Liaoning Province, P. R. China.
}

\begin{abstract}
The ever-increasing demand for propene has driven some new strategies to produce propene, such as propane dehydrogenation, metathesis of ethene and 2-butene, catalytic cracking of $\mathrm{C}_{4}$ alkenes, conversion of methanol and ethanol, and direct conversion of ethene, instead of the conventional naphtha-cracking process. The transformation of ethene to propene (ETP) is of great interest owing to the abundant supply of ethylene from ethane crackers and shale gas recently. HZSM-5 zeolite is an effective ETP catalyst and the acid properties of HZSM-5 significantly affect ethene conversion and propene
\end{abstract}

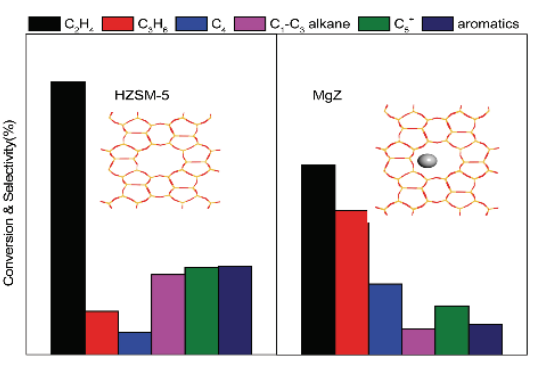
selectivity. A series of HZSM-5 zeolites modified with different amounts of $\mathrm{Mg}(0-1.0 \%)$ were prepared by an incipient impregnation method. Their structures and acidity were systematically characterized by X-ray diffraction (XRD), $\mathrm{N}_{2}-$ adsorption/desorption analysis, ${ }^{27} \mathrm{Al}$ magic-angle spinning nuclear-magnetic-resonance $\left({ }^{27} \mathrm{Al}\right.$ MAS NMR), ${ }^{29} \mathrm{Si}$ MAS NMR, temperature-programmed desorption of $\mathrm{NH}_{3}\left(\mathrm{NH}_{3}-\mathrm{TPD}\right)$, and Fourier transform infrared (FT-IR) of pyridine adsorption techniques. Their catalytic performances in the direct conversion of ethene to propene using a continuous-flow fixed-bed micro-reactor were evaluated. The effects of reaction conditions and $\mathrm{Mg}$ contents were thoroughly investigated. Ethene conversion is found to decrease with increasing reaction temperature and gaseous hourly space velocity (GHSV) of HZSM5 zeolite, while the selectivities of propene show the opposite trend. The optimized reaction temperature is $550{ }^{\circ} \mathrm{C}$ and GHSV is $3000 \mathrm{~h}^{-1}$ for the maximum propene yield. After Mg modification, ethene conversion decreases, while propene selectivity increases for HZSM-5 with increasing Mg loading under the optimized reaction conditions. HZSM-5 modified with an appropriate amount of $0.5 \% \mathrm{Mg}$ shows enhanced selectivity of propene exceeding $45 \%$, and the propene yield is maintained above $20 \%$. Moreover, the selectivity of by-product aromatics is less than $8 \% .{ }^{27} \mathrm{Al}$ and ${ }^{29} \mathrm{Si}$ MAS NMR results indicate that the introduction of $\mathrm{Mg}$ will result in dealumination in HZSM-5. $\mathrm{NH}_{3}$-TPD and pyridine-IR results indicate that the addition of $\mathrm{Mg}$ will reduce both the total acid amount and strong Brønsted acid amount and that the proper amount of Mg leads to increase in the amount of medium-strong acid. By-products such as alkanes and aromatics are mainly formed at these strong Brønsted acid sites by oligomerization and hydrogen transfer reactions. Therefore, the introduction of $\mathrm{Mg}$ decreases the number of strong Brønsted acid sites and further enhances the selectivity of propene. Temperatureprogrammed oxidation (TPO) and ${ }^{13} \mathrm{C}$ CP/MAS NMR analysis of the coked catalysts indicate that the addition of Mg not only inhibits coke deposition, but also changes the type of coke. However, excessive Mg modification results in a remarkable reduction of HZSM-5 activity owing to the significant decrease in the number of total acid and strong Brønsted acid sites.

Key Words: HZSM-5 zeolite; Mg modification; Acidity; Propene; ETP reaction

Received: October 12, 2017; Revised: October 31, 2017; Accepted: November 3, 2017; Published online: November 10, 2017.

${ }^{*}$ Corresponding author. Email: wpzhang@dlut.edu.cn; Tel: +86-411-84986326.

The project was supported by the National Natural Science Foundation of China $(21373035,21673027)$ and the Fundamental Research Funds for the Central Universities in China (DUT16RC(3)002, DUT17TD04).

国家自然科学基金(21373035, 21673027)及中央高校基本科研业务费专项资金(DUT16RC(3)002, DUT17TD04)项目资助

(C) Editorial office of Acta Physico-Chimica Sinica 


\title{
镁改性对 HZSM-5 分子篮催化乙烯制丙烯的影响
}

徐禄禄, 赵侦超, 赵蓉蓉, 喻瑞, 张维萍 ${ }^{*}$

大连理工大学化工学院, 精细化工国家重点实验室, 辽宁 大连 116024

\begin{abstract}
摘要：采用等体积浸渍法制备了一系列不同 Mg含量(0-1.0\%)的HZSM-5分子篮。利用X射线衍射 $(X R D) 、 N_{2}$ 吸附/脱附、 铝魔角旋转固体核磁共振 $\left({ }^{27} \mathrm{Al}\right.$ MAS NMR)、 ${ }^{29} \mathrm{Si}$ MAS NMR、氨-程序升温脱附 $\left(\mathrm{NH}_{3}-\mathrm{TPD}\right)$ 和吡啶吸附傅里叶变换红外 (Pyridine-IR)光谱等技术对改性前后样品的结构和酸性进行了详细表征, 在常压连续流动固定床反应器上考察其对乙烯 转化制丙烯(ETP)反应的催化性能, 评价了反应条件和Mg改性的影响。结果表明, 在温度为 $550^{\circ} \mathrm{C}$ 、乙烯体积空速 $\mathrm{GHSV}=$ $3000 \mathrm{~h}^{-1}$ 的适宜反应条件下, $0.5 \%$ 适量镁改性HZSM- 5 导致乙烯转化率有所下降, 但丙烯选择性增加到 $45 \%$ 以上, 而副

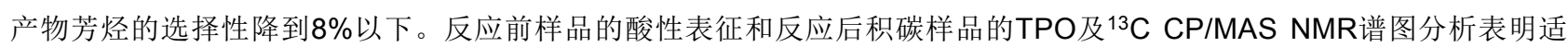
量镁改性使HZSM-5分子笁的总酸量和强B酸量减少而中强酸量增加, 从而提高了丙烯的选择性, 但是过量的镁改性使分 子篮的总酸量明显减少, 导致催化剂的活性显著下降。
\end{abstract}

关键词: HZSM-5分子篮; 镁改性; 酸性; 丙烯; 乙烯制丙烯反应 中图分类号: 0643.3

\section{1 引言}

丙烯是石油化工行业最重要的基本有机原料 之一, 主要用于生产聚丙烯、丙烯腈、环氧丙烷、 异丙醇等化工产品。中国的丙烯需求年均增长达 到 $6.3 \%$, 其中聚丙烯占丙烯衍生物市场的 $90 \%$ 。 目前生产丙烯主要有丙烷脱氢 1-3、乙烯和 2-丁烯 歧化 ${ }^{4-6}$ 、合成气制烯烃 7 、甲醇转化 ${ }^{8-11}$ 等技术。 而由乙烯出发催化转化制丙烯(ETP)的路线最近 引起人们的广泛关注, 该路线对于解决乙烯产能 过剩也具有重要意义。目前用于 ETP 反应的催化 剂主要集中在具有八元环的 SSZ-13、SAPO-34 分子 篮 ${ }^{12-15}$ 和十元环的 ZSM-5 分子篮 ${ }^{16,17}$, 而金属负载 型介孔材料 MCM-41 催化剂也有少量报道 18,19 。分 子篮催化剂由于具有可调节的酸性和孔道结构以 及良好的稳定性, 在 ETP 反应中应用前景广泛。 Dai 等人 12 比较了具有相似硅铝比而不同孔径大 小的分子篮催化剂用于 ETP 反应, 发现八元环的 HSSZ-13 沸石的反应性能明显优于十元环的 HZSM-5。在甲醇制丙烯的研究中, HZSM-5 分子 篮由于其较高的丙烯选择性和良好的抗积炭失活 性能, 目前成为甲醇制丙烯的首选催化剂 7-9,20-22。 其合适的孔道结构对 ETP 反应也至关重要, Liu 等 人 16 就认为在所测试的 11 种分子篮中 HZSM-5 的 ETP 反应性能最好。除此之外, 分子篮的酸性也 影响丙烯的选择性。一般认为在酸催化下, 乙烯聚 合产生高碳烯烃, 生成的高碳烯烃进一步裂解产 生目的产物丙烯, 过程中生成的低碳烯烃通过缩 聚、氢转移和芳构化等副反应生成高碳烯烃、烷烃
和芳烃, 这样在降低目的产物选择性的同时还因 积炭导致催化剂活性迅速下降 15,23-25。在 ETP 反 应中, 分子篎酸性对催化性能的影响研究不多。其 中磷或硼改性 HZSM-5 可以提高 ETP 反应中低碳 烯烃的收率 ${ }^{16}$, 而镁改性 HZSM-5 在甲醇制丙烯 反应中能提升丙烯收率和催化剂稳定性 ${ }^{21}$, 但在 ETP 反应中没有报道。本文通过硝酸镁浸渍改性 硅铝原子比为 25 左右的 HZSM-5 分子篎来调节其 酸量和酸强度的分布, 利用 XRD、 $\mathrm{N}_{2}$ 吸附-脱附、 固体 NMR、 $\mathrm{NH}_{3}$-TPD 和 pyridine-IR 等方法系统 考察了镁改性前后催化剂的结构特征和酸性变化 及其与 ETP 催化反应性能的关联。

\section{2 实验部分}

\section{1 催化剂制备}

$\mathrm{NH}_{4}-\mathrm{ZSM}-5$ 分子篮 $(\mathrm{Si} / \mathrm{Al}=25)$ 由南开大学购 买, 在马弗炉中 $550^{\circ} \mathrm{C}$ 焙烧 $5 \mathrm{~h}$ 得到 HZSM-5。 采用等体积浸渍法制取一系列不同 $\mathrm{Mg}$ 含量的 HZSM-5 样品, 经 $110^{\circ} \mathrm{C}$ 烘干再于 $550^{\circ} \mathrm{C}$ 马弗炉 中焙烧 $5 \mathrm{~h}$, 即得到不同 $\mathrm{Mg}$ 负载量的 $\mathrm{Mg} / \mathrm{HZSM}$ 5 分子篎, 记为 MgZ-x, $x$ 代表 HZSM-5 分子篎中 $M g$ 的质量含量 $(\%)$, 由 ICP 方法测得。

\section{2 催化剂表征}

样品的晶相结构在 PANalytical X'Pert 3 型粉 末 $X$ 射线衍射仪上确定 $(\mathrm{Cu}$ 靶, 管压 $40 \mathrm{kV})$, 扫 描角度为 $5^{\circ}-50^{\circ}$, 扫描速率 $5\left(^{\circ}\right) \cdot \mathrm{min}^{-1}$ 。相对结晶 度用样品的特征峰 $\left(2 \theta=22.5^{\circ}-25^{\circ}\right)$ 面积与参比样 的特征峰面积的比值表示, 以未改性的 HZSM-5 
样品作为参比样 ${ }^{22,26}$ 。 $\mathrm{Mg}$ 的负载量由 Perkin Elemer 公司生产的型号为 Optima 2000DV 的电感 耦合等离子体质谱仪(ICP-MS)得到。

$\mathrm{N}_{2}$ 吸附-脱附实验在 Micromeritics ASAP 2000 上进行。样品置于 $350{ }^{\circ} \mathrm{C}$ 下抽空预处理 $6 \mathrm{~h}$, 然后 在液氮温度下进行吸脱附, 用 BET 方程计算样品 的总比表面积, $t$-plot 法计算样品的外比表面积。

固体核磁共振实验均在 Agilent DD2-500 $\mathrm{MHz}$ 谱仪上进行。 ${ }^{27} \mathrm{Al}$ MAS NMR 采用 $4 \mathrm{~mm}$ 探 头, 共振频率是 $130.2 \mathrm{MHz}, \pi / 12$ 脉冲宽度为 0.3 $\mu \mathrm{s}$, 弛豫延迟为 $2 \mathrm{~s}, 200$ 次累加, 样品转速为 14 $\mathrm{kHz}$, 化学位移参考为 $1 \% \mathrm{Al}\left(\mathrm{NO}_{3}\right)_{3}$ 水溶液。 ${ }^{29} \mathrm{Si}$ MAS NMR采用 $6 \mathrm{~mm}$ 探头, 共振频率为 $99.3 \mathrm{MHz}$, 采用高功率质子去偶, $\pi / 4$ 脉宽 $2.8 \mu \mathrm{s}$, 弛豫延迟 为 $4 \mathrm{~s}, 500$ 次累加, 转速为 $4 \mathrm{kHz}$, 化学位移参考 为四甲基硅烷。 ${ }^{13} \mathrm{C} \mathrm{CP} / \mathrm{MAS} \mathrm{NMR}$ 采用 $4 \mathrm{~mm}$ 探 头, 共振频率为 $125.7 \mathrm{MHz}$, 接触时间为 $4 \mathrm{~ms}$, 弛 豫延迟为 $4 \mathrm{~s}, 1000$ 次累加, 转速为 $10 \mathrm{kHz}$, 化学 位移参考为四甲基硅烷。采用 DMFit 软件及合适 的 Gaussian-Lorentzian 线型拟合谱图。 ${ }^{27} \mathrm{Al} \mathrm{MAS}$ NMR 测试前样品均置于含有硝酸铵水溶液的干 燥器中饱和吸水, 使固体核磁共振获得最大程度 可见铝。

$\mathrm{NH}_{3}$ 程序升温脱附(TPD) 实验在自制装置上 进行, 以高纯 $\mathrm{N}_{2}$ 为载气, 样品先在 $\mathrm{He}$ 中预处理 $30 \mathrm{~min}$, 然后冷却至 $100{ }^{\circ} \mathrm{C}$ 吸附 $5 \% \mathrm{NH}_{3} / \mathrm{N}_{2}$ 至饱 和, 经 $\mathrm{N}_{2}$ 吹扫以除去物理吸附的 $\mathrm{NH}_{3}$ 后, 以 $10^{\circ} \mathrm{C} \cdot \mathrm{min}^{-1}$ 的升温速率进行脱附, 脱附出来的 $\mathrm{NH}_{3}$ 由 OMNIstar GSD-320 型质谱仪测定。

吸附吡啶的红外光谱表征在 Bruker VERTEX70 傅里叶变换红外(FT-IR)光谱仪上进 行, 仪器的分辨率 $0.4 \mathrm{~cm}^{-1}$, 扫描次数 32 。样品先 在 $400^{\circ} \mathrm{C}$ 脱附 $2 \mathrm{~h}$, 降至室温吸附吡啶, 然后分别 升至 $150{ }^{\circ} \mathrm{C} 、 300^{\circ} \mathrm{C}$ 进行脱附后测试谱图。

程序升温氧化 (TPO) 实验在自制装置上进 行, 样品在 $10 \% \mathrm{O}_{2} / \mathrm{He}$ 气体中以 $10{ }^{\circ} \mathrm{C} \cdot \mathrm{min}^{-1}$ 的速 率从 $100{ }^{\circ} \mathrm{C}$ 升到 $800{ }^{\circ} \mathrm{C}$, 以质谱仪(OMNIstar GSD-320)连续检测尾气的 $\mathrm{CO}_{2}$ 的信号曲线。

\section{3 催化剂反应性能评价}

采用常压连续流动微型固定床反应器来评价 催化剂的反应性能。催化剂用量为 $0.1 \mathrm{~g}$, 在高纯 $\mathrm{N}_{2}$ 气氛下程序升温至 $450^{\circ} \mathrm{C}$ 进行预处理以除去物 理吸附的杂质, 改变温度至反应温度后, 通入乙烯 进入催化剂床层反应, 产物采用 Shimadzu GC2014C 型气相色谱进行在线分析, HP-Plot-Q 毛细 管柱, 氢火焰离子化检测器(FID)。反应器出口至
色谱仪进样口的连接管用保温带保持 $160{ }^{\circ} \mathrm{C}$ 以避 免产物冷凝。采用碳数平衡法计算乙烯的转化率 和产物的选择性, 公式如下:

$$
\begin{aligned}
& \text { Conv }=\frac{\sum_{i} A_{i}}{\sum_{i} A_{i}+A_{\text {ethene }}} \times 100 \% \quad \text { (乙烯的转化率) } \\
& \operatorname{Sel}_{i}=\frac{A_{i}}{\sum_{i} A_{i}} \times 100 \% \quad \text { (产物的选择性) } \\
& (A: \text { 色谱出峰面积) }
\end{aligned}
$$

\section{3 结果与讨论}

\section{$3.1 X R D$ 和 $\mathrm{N}_{2}$ 吸附/脱附表征}

图 1 给出了镁改性前后 HZSM-5 的 XRD 谱 图。由图可以看出, HZSM-5的结晶度较高, 具有 典型的MFI结构 26 。负载Mg后, HZSM-5 的主要衍 射峰位置没有变化, 说明 $\mathrm{Mg}$ 改性并未对分子篮的 长程有序晶体结构产生明显影响。以未改性的 HZSM-5作为参比样计算的相对结晶度见表 1 , 随 着负载 $\mathrm{Mg}$ 量的增加, 样品的相对结晶度逐渐减 小, 特别是 $\mathrm{Mg}$ 含量为 $1 \%$ 时, HZSM- 5 的相对结晶 度下降到 $72 \%$ 。这可能是 $\mathrm{Mg}$ 的大量加入使HZSM5分子篮的局部结构有所改变。除此之外, 没有发 现晶形 $\mathrm{MgO}$ 衍射峰产生, 说明 $\mathrm{MgO}$ 在分子篮上是 高度分散的。

镁改性前后 HZSM-5 分子篮的比表面积和孔 体积结果见表 1 。可以看出, 随着 $\mathrm{Mg}$ 含量的增加, 样品的总比表面积和外比表面积均减少, 其中外 比表面积下降多一些。当 $\mathrm{Mg}$ 含量比较低时, 样品 的外比表面积变化不是很明显, 总比表面积下降 主要是由内比表面积下降引起的, 随着 $\mathrm{Mg}$ 含量 增加, 外比表面积下降越来越明显, 这表明低含量 的 $\mathrm{Mg}$ 改性可能主要在内表面, $\mathrm{Mg}$ 含量提高后, 氧化镁沉积在内表面同时也可能沉积在外表面。

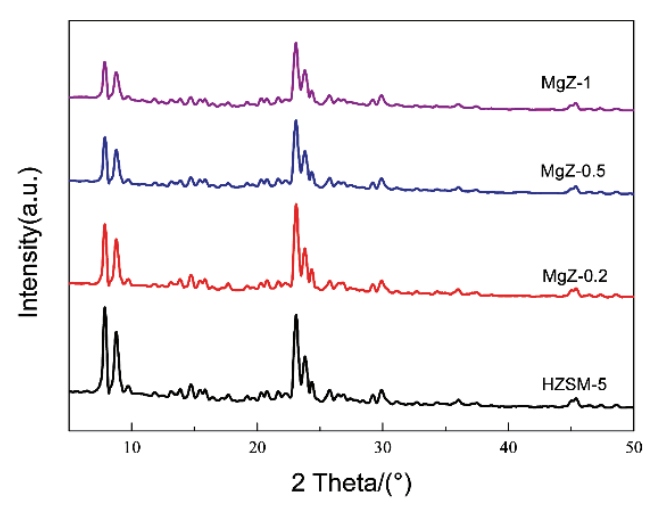

图 1 不同 Mg 含量 HZSM-5 分子篮的 XRD 谱

Fig. 1 XRD patterns of HZSM-5 zeolites modified with different Mg contents. 
表 1 不同 Mg 改性 HZSM-5 分子笁的相对结晶度、比表面积、孔容、硅铝比以及硅羟基含量

Table 1 The relative crystallinitines, surface areas, pore volumes, $\mathrm{Si} / \mathrm{Al}$ ratios and $\mathrm{SiOH}$ amounts of HZSM-5 zeolites modified by different Mg contents.

\begin{tabular}{|c|c|c|c|c|c|c|}
\hline \multirow[b]{2}{*}{ Sample } & \multirow[b]{2}{*}{ Relative crystallinity $/ \%$} & \multicolumn{2}{|c|}{$S_{\mathrm{BET}} /\left(\mathrm{m}^{2} \cdot \mathrm{g}^{-1}\right)$} & \multirow[b]{2}{*}{$V_{\text {pore }} /\left(\mathrm{cm}^{3} \cdot \mathrm{g}^{-1}\right)$} & \multirow[b]{2}{*}{$\mathrm{Si} / \mathrm{Al}$ ratio ${ }^{\mathrm{b}}$} & \multirow[b]{2}{*}{$\mathrm{SiOH}$ content $/ \%$} \\
\hline & & total & external $^{\mathrm{a}}$ & & & \\
\hline HZSM-5 & 100 & 374 & 98 & 0.18 & 24 & 2.6 \\
\hline $\mathrm{MgZ}-0.2$ & 91 & 356 & 94 & 0.18 & 30 & 3.8 \\
\hline MgZ-0.5 & 81 & 349 & 85 & 0.21 & 34 & 5.3 \\
\hline MgZ-1 & 72 & 345 & 71 & 0.20 & 40 & 6.1 \\
\hline
\end{tabular}

${ }^{a}$ from t-plot method, ${ }^{b-c}$ from ${ }^{29}$ Si MAS NMR measurements.

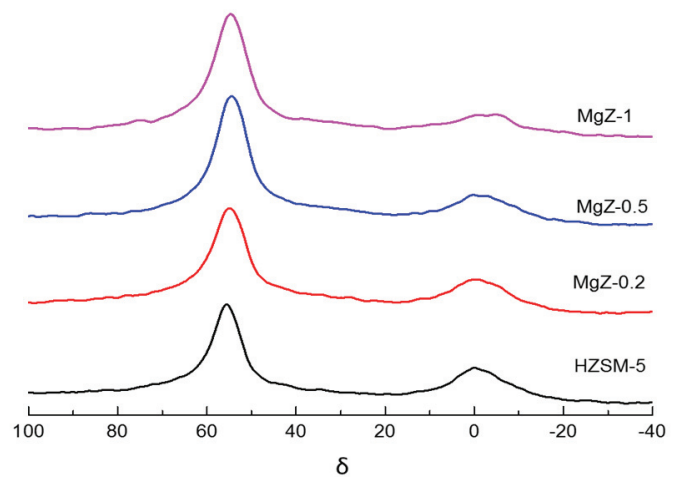

图 2 不同 Mg 含量 HZSM-5 分子篮的 ${ }^{27} \mathrm{Al}$ MAS NMR 谱

Fig. $2{ }^{27}$ Al MAS NMR spectra of HZSM-5 zeolites modified with different $\mathrm{Mg}$ contents.

另外, $\mathrm{Mg}$ 改性后并没有堵塞 HZSM-5 的孔道, 致 使其孔体积下降不明显。

\section{2 ${ }^{27}$ AI MAS NMR 和 ${ }^{29}$ Si MAS NMR 表征}

${ }^{27} \mathrm{Al}$ MAS NMR谱可以用来确定分子篮中铝 物种的配位状态。图2是镁改性前后HZSM-5分子 耖的 ${ }^{27} \mathrm{Al}$ MAS NMR 谱图。主要在 $\delta=54$ 和 $\delta=0$ 处

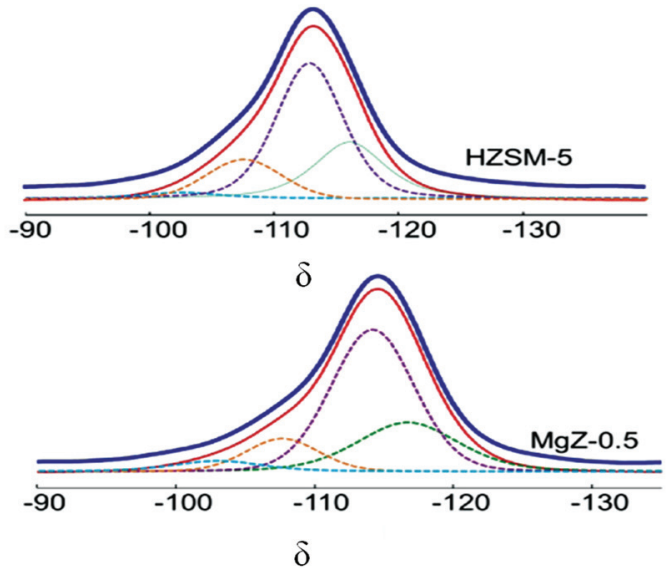

有两处峰, 其中前者归属为四配位的骨架铝, 后者 归属为六配位的非骨架铝物种 26,27 。图2可知改性 前后样品中主要存在四配位骨架铝，与未改性的 HZSM-5相比, 改性后样品骨架铝强度看上去没有 明显下降，而非骨架铝强度看上去略有减少。

不同 $\mathrm{Mg}$ 改性 HZSM-5 分子篮的 ${ }^{29} \mathrm{Si}$ MAS NMR 谱如图 3 所示。化学位移在 $\delta=-116$ 和 $\delta=$ -112 处的峰归属为 HZSM-5 分子耖中两个结晶学 不等价位的 $\mathrm{Si}(\mathrm{OSi})_{4}$ 基团, $\delta=-106$ 处的峰归属于 $\mathrm{Si}(\mathrm{OAl})(\mathrm{OSi})_{3}$ 基团, 而 $\delta=-103$ 处的峰归属为硅 着基即 $\mathrm{Si}(\mathrm{OH})(\mathrm{OSi})_{3}$ 基团 ${ }^{26}$ 。对不同 $\mathrm{Mg}$ 改性 HZSM-5 分子䇻 ${ }^{29} \mathrm{Si}$ MAS NMR 谱峰进行定量拟 合可以得到分子筛的骨架 $\mathrm{Si} / \mathrm{Al}$ 比(表 1)。从表中 可以看出, 随着 $\mathrm{Mg}$ 含量的增加, 分子笁的骨架 $\mathrm{Si} / \mathrm{Al}$ 比有增加, 这说明 $\mathrm{Mg}$ 改性后分子篎存在一 定程度的脱铝，同时导致硅羟基的含量也增加了。 特别是 $1 \% \mathrm{Mg}$ 改性的 HZSM-5 分子篮的 Si/Al 比 和硅羟基含量增加明显, 表明该样品脱铝显著导 致局部结构破坏。这与前面 XRD 和 BET 比表面 积测试结果一致。但该样品的 ${ }^{27} \mathrm{Al}$ MAS NMR 谱

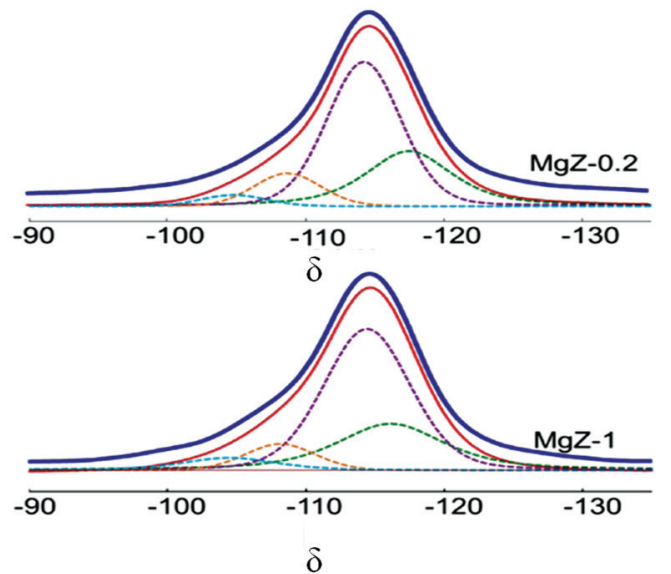

$\delta$

图 3 不同 Mg 改性 HZSM-5 分子笁的 ${ }^{29} \mathrm{Si}$ MAS NMR 谱及其定量拟合谱

Fig. $3{ }^{29}$ Si MAS NMR and quantitatively deconvoluted spectra of HZSM-5 zeolites modified with different Mg contents. 

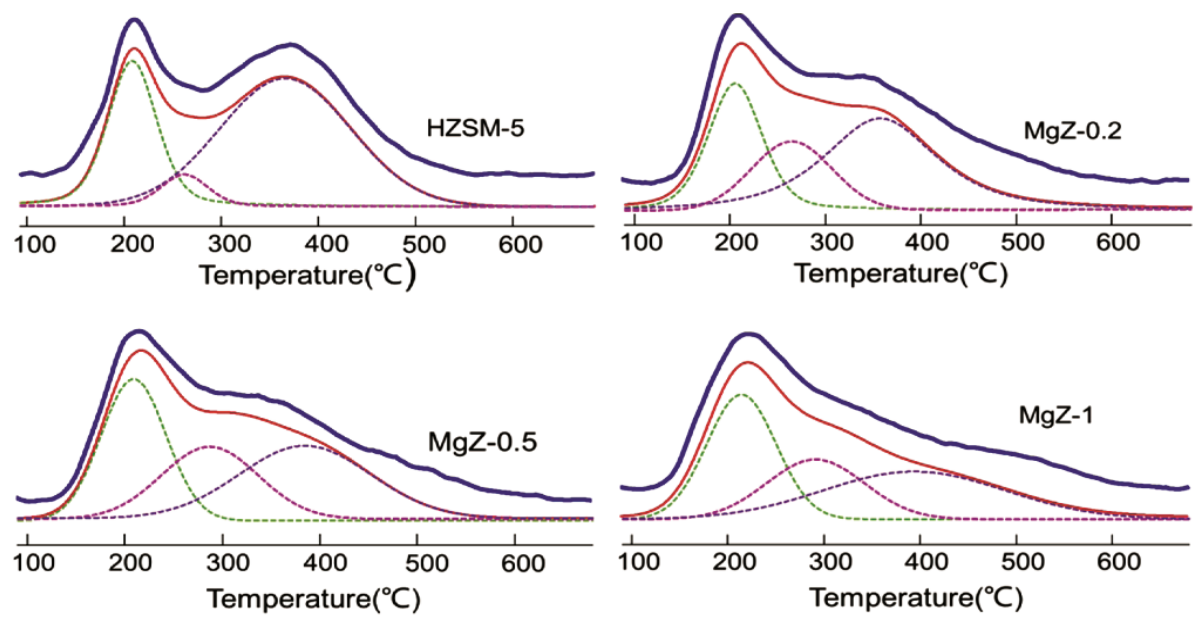

图 4 不同 Mg 改性 HZSM-5 分子篮的 $\mathrm{NH}_{3}-\mathrm{TPD}$ 曲线

Fig. $4 \mathrm{NH}_{3}$-TPD profiles of HZSM-5 zeolites modified with different Mg contents.

表 2 不同 Mg 改性 HZSM-5 分子篎的酸性变化

Table 2 Acidity variations of HZSM-5 zeolites modified with different Mg contents.

\begin{tabular}{|c|c|c|c|c|c|c|c|c|c|c|}
\hline \multirow[t]{2}{*}{ Sample } & \multirow{2}{*}{$\begin{array}{c}\text { Total acid } \\
\text { amount } /\left(\mathrm{mmol}^{\prime} \mathrm{g}^{-1}\right)^{\mathrm{a}}\end{array}$} & \multicolumn{3}{|c|}{$\begin{array}{l}\text { Quantity of acid } \\
\text { sites } /\left(\mathrm{mmol}^{\circ} \mathrm{g}^{-1}\right)^{\mathrm{a}}\end{array}$} & \multicolumn{2}{|c|}{$\begin{array}{c}\text { Brønsted acid } \\
\text { sites } /\left(\mathrm{mmol} \cdot \mathrm{g}^{-1}\right)^{\mathrm{b}}\end{array}$} & \multicolumn{2}{|c|}{ Lewis acid sites $/\left(\mathrm{mmol}^{-\mathrm{g}^{-1}}\right)^{\mathrm{b}}$} & \multicolumn{2}{|c|}{$N_{\mathrm{B}} / N_{\mathrm{L}}^{\mathrm{b}}$} \\
\hline & & strong & middle & weak & $150^{\circ} \mathrm{C}$ & $300^{\circ} \mathrm{C}$ & $150^{\circ} \mathrm{C}$ & $300^{\circ} \mathrm{C}$ & $150^{\circ} \mathrm{C}$ & $300^{\circ} \mathrm{C}$ \\
\hline HZSM-5 & 0.57 & 0.37 & 0.04 & 0.16 & 0.39 & 0.16 & 0.15 & 0.04 & 2.6 & 4.0 \\
\hline MgZ-0.2 & 0.51 & 0.25 & 0.11 & 0.15 & 0.33 & 0.14 & 0.30 & 0.08 & 1.1 & 1.8 \\
\hline MgZ-0.5 & 0.41 & 0.15 & 0.11 & 0.15 & 0.29 & 0.08 & 0.29 & 0.07 & 1.0 & 1.1 \\
\hline MgZ-1 & 0.35 & 0.12 & 0.09 & 0.14 & 0.20 & 0.04 & 0.24 & 0.06 & 0.8 & 0.7 \\
\hline
\end{tabular}

${ }^{a}$ from $\mathrm{NH}_{3}$-TPD measurements, ${ }^{\mathrm{b}}$ from pyridine-IR measurements.

图没有出现明显的非骨架铝含量增加。这可能是 由于添加的 $\mathrm{Mg}$ 与分子篮中的铝物种相互作用使 得铝的局部环境发生变化从而出现核磁共振不可 见铝 26,28 。

\section{3 $\mathrm{NH}_{3}$-TPD 表征}

图 4 是 $\mathrm{Mg}$ 改性前后 HZSM-5 分子篮样品的 $\mathrm{NH}_{3}$-TPD曲线。对于改性前后的HZSM-5分子耖, $\mathrm{NH}_{3}$-TPD谱图上均有三个明显的脱附峰。其中根 据脱附温度由低到高依次归属为 HZSM-5 上弱 酸、中强酸和强酸位上的 $\mathrm{NH}_{3}$ 脱附信号。由脱附峰 温可以判断催化剂酸性的强弱, 由脱附峰面积可 以判断催化剂酸量的大小 22,29 。样品的总酸量以及 各种酸性分布数量列于表 2 。从中可以看出, $\mathrm{Mg}$ 改 性对分子節的酸密度和酸性分布有一定程度的影 响。随着负载镁的增加HZSM-5分子篎的总酸密度 逐渐减少, 三种不同强度的酸位数量变化不尽相 同。随着 $\mathrm{Mg}$ 含量的增加, HZSM-5上强酸数量明显 减少, 中强酸的数量呈现先增加后减少的趋势, 而 弱酸的数量基本保持不变。这表明Mg改性HZSM5 时, 主要是覆盖了强酸位, 过量 $M g$ 的加入也会覆 盖中强酸位, 而对弱酸位的影响不大。

\section{4 吸附吡啶的红外光谱表征}

图 5为HZSM-5与镁改性后MgHZSM-5吸附吡 啶并在 $300{ }^{\circ} \mathrm{C}$ 脱附后的红外光谱图。1540和 1450 $\mathrm{cm}^{-1}$ 附近处的信号分别为吡啶吸附在 $\mathrm{B}$ 酸中心和 $\mathrm{L}$ 酸中心形成的吸收峰, $1490 \mathrm{~cm}^{-1}$ 处的信号为吡啶

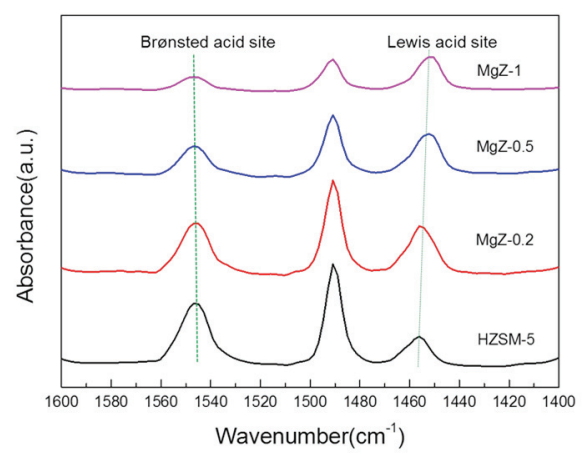

图 5 不同 Mg 改性 HZSM-5 分子篮吸附吡啶并 经 $300{ }^{\circ} \mathrm{C}$ 脱附的 pyridine-IR 光谱

Fig. 5 Pyridine-IR spectra of pyridine adsorbed on the HZSM-5 zeolites modified with different Mg contents after desorption at $300{ }^{\circ} \mathrm{C}$. 
吸附在 $\mathrm{B}$ 酸中心和 $\mathrm{L}$ 酸中心共同形成的吸收峰 ${ }^{30}$ 。 每个样品中 B酸和 $\mathrm{L}$ 酸的量及两者比值 $N_{\mathrm{B}} / N_{\mathrm{L}}$ 的计 算值均列于表 2 。可以看出, 在未改性的样品中, $\mathrm{B}$ 酸位的数量明显多于 L酸位的数量, 而且强酸中 以 $\mathrm{B}$ 酸为主。改性后的样品随着负载 $\mathrm{Mg}$ 含量的增 加, B酸数量不断在减少。结合 $\mathrm{NH}_{3}-\mathrm{TPD}$ 结果可 知, $\mathrm{Mg}$ 改性后减少的强酸以 $\mathrm{B}$ 酸为主, 而且从 $\mathrm{B}$ 酸 与 $\mathrm{L}$ 酸数量比值看出, $1.0 \% \mathrm{Mg}$ 含量的样品酸中心 变为以 L酸为主, 这说明镁改性后覆盖了一部分 $\mathrm{B}$ 酸位。改性前后样品的L酸数量呈现先增加后减少 的趋势, 并且从 $1455 \mathrm{~cm}^{-1}$ 移向 $1450 \mathrm{~cm}^{-1}$ 处(图5中 所标坚线)。Ward ${ }^{31}$ 认为 $1450 \mathrm{~cm}^{-1}$ 出现吸收峰可以 归因为 $\mathrm{Mg}(\mathrm{OH})^{+}$, 该物种来源于 $\mathrm{Mg}^{2+}$ 与 $\mathrm{B}$ 酸位的桥 差基作用产生的。而随着生成 $\mathrm{Mg}(\mathrm{OH})^{+}$的增加, $\mathrm{Mg}(\mathrm{OH})^{+}$自行缩聚生成 $\mathrm{MgO}$, 所以 $\mathrm{L}$ 酸先增加后减 少。以上说明, Mg改性对HZSM-5分子耖酸中心的 类型和数量均有影响, $0.5 \% \mathrm{Mg}$ 改性的HZSM-5具 有适当含量的强B酸和中强酸中心。

\section{5 催化反应性能}

\subsection{1 反应温度和空速的影响}

表 3 为HZSM-5 分子篮在不同温度下乙烯制丙 烯在反应 $7 \mathrm{~h}$ 时的结果。从中可以看出, 随着反应 温度升高, 乙烯的转化率逐渐降低而丙烯选择性 增加。由于整个反应过程中存在高碳烃裂解, 所以 温度升高对丙烯的选择性增加, 进而丙烯产率由 初始的 $8 \%$ 增加到 $14 \%$ 。这与Dai等人 ${ }^{12}$ 使用 SSZ-13 分子笁催化ETP反应结果一致。但同时温度过高也 会加剧烯烃聚合生成长链烃和芳烃等副产物最终
以积碳形式堵塞孔道和活性位。因此当反应温度 为 $600{ }^{\circ} \mathrm{C}$ 时, 虽然丙烯产率还保持较高, 但乙烯转 化率下降过快。故最佳反应温度为 $550{ }^{\circ} \mathrm{C}$ 。表 4 为 HZSM-5 分子篮在不同空速下乙烯制丙烯的反应 结果。从中可知, 空速增加乙烯的转化率随之下 降, 而丙烯的选择性由 $18 \%$ 提高到 $39 \%$ 。这是因为 空速增加反应物乙烯与催化剂活性位接触时间变 短, 抑制了初次反应产物进行二次反应。因此适宜 的乙烯反应空速为 $3000 \mathrm{~h}^{-1}$ 。

\subsection{2 镁改性对 ETP 反应性能的影响}

在常压 $550{ }^{\circ} \mathrm{C}$ 、乙烯体积空速(GHSV)为 3000 $\mathrm{h}^{-1}$ 的适宜条件下，考察了不同镁改性对 ETP反应 性能的影响。图6列出了乙烯在 $\mathrm{Mg}$ 改性前后 HZSM-5 分子篮上的转化率和主要产物的选择 性。可以看出(1) 乙烯在 $\mathrm{Mg}$ 改性后催化剂上的反 应活性明显低于未改性的催化剂, 在 $\mathrm{Mg}$ 含量 $1 \%$ 时 乙烯转化率由初始的 $85 \%$ 降至 $30 \%$ 左右，这表明 Mg改性覆盖了酸性位导致反应活性降低; (2) Mg 改性后HZSM-5催化剂上目的产物丙烯和 $\mathrm{C}_{4}$ 的选 择性明显提高, 而且随着 $\mathrm{Mg}$ 负载量的增加, 选择 性逐渐增加。其中 $0.5 \%-1 \% \mathrm{Mg} / \mathrm{HZSM}-5$ 对丙烯的 选择性达到 $45 \%-54 \%$, 高于Follmanna等人 ${ }^{17}$ 报道 的HZSM-23 和HZSM-5 分子篮上ETP反应中丙烯 选择性。另外, 随着 $\mathrm{Mg}$ 含量增加到 $0.5 \%$ 在HZSM5 上烷烃选择性降至 $15 \%$ 以下, 芳烃选择性降至 $8 \%$, 这说明 $\mathrm{Mg}$ 改性覆盖的酸性位抑制了低碳烃聚 合及氢转移等副反应发生; (3) 由于 $0.2 \% \mathrm{Mg}$ 改性 样品中 $\mathrm{Mg}$ 含量较低, 覆盖的酸性位少, 所以虽然

表 3 不同反应温度下 HZSM-5 分子笁上乙烯制丙烯的催化性能

Table 3 Catalytic performances of HZSM-5 zeolite for ETP at different temperatures.

\begin{tabular}{|c|c|c|c|c|c|c|c|}
\hline \multirow{2}{*}{ Temperature $/{ }^{\circ} \mathrm{C}$} & \multirow{2}{*}{$\mathrm{C}_{2} \mathrm{H}_{4}$ conversion $/ \%$} & \multicolumn{5}{|c|}{ Product selectivity $/ \%$} & \multirow{2}{*}{$\mathrm{C}_{3} \mathrm{H}_{6}$ yield $/ \%$} \\
\hline & & $\mathrm{C}_{3} \mathrm{H}_{6}$ & $\mathrm{C}_{4} \mathrm{H}_{8}$ & $\mathrm{C}_{1}-\mathrm{C}_{4}$ alkane & $\mathrm{C}_{5}^{+}$ & aromatics & \\
\hline 450 & 94 & 9 & 24 & 29 & 21 & 17 & 8 \\
\hline 500 & 89 & 13 & 16 & 30 & 21 & 20 & 12 \\
\hline 550 & 81 & 18 & 5 & 32 & 23 & 22 & 15 \\
\hline 600 & 36 & 39 & 9 & 15 & 22 & 15 & 14 \\
\hline
\end{tabular}

表 4 不同空速下 HZSM-5 分子䈨上乙烯制丙烯的催化性能

Table 4 Catalytic performances of HZSM-5 zeolite for ETP at different GHSVs.

\begin{tabular}{|c|c|c|c|c|c|c|c|}
\hline \multirow{2}{*}{$\mathrm{GHSV} / \mathrm{h}^{-1}$} & \multirow{2}{*}{$\mathrm{C}_{2} \mathrm{H}_{4}$ conversion $/ \%$} & \multicolumn{5}{|c|}{ Product selectivity $/ \%$} & \multirow{2}{*}{$\mathrm{C}_{3} \mathrm{H}_{6}$ yield $/ \%$} \\
\hline & & $\mathrm{C}_{3} \mathrm{H}_{6}$ & $\mathrm{C}_{4} \mathrm{H}_{8}$ & $\mathrm{C}_{1}-\mathrm{C}_{4}$ alkane & $\mathrm{C}_{5}^{+}$ & aromatics & \\
\hline 1400 & 81 & 18 & 5 & 32 & 23 & 22 & 15 \\
\hline 3000 & 61 & 39 & 10 & 21 & 17 & 13 & 24 \\
\hline 3600 & 56 & 39 & 12 & 20 & 17 & 12 & 22 \\
\hline
\end{tabular}




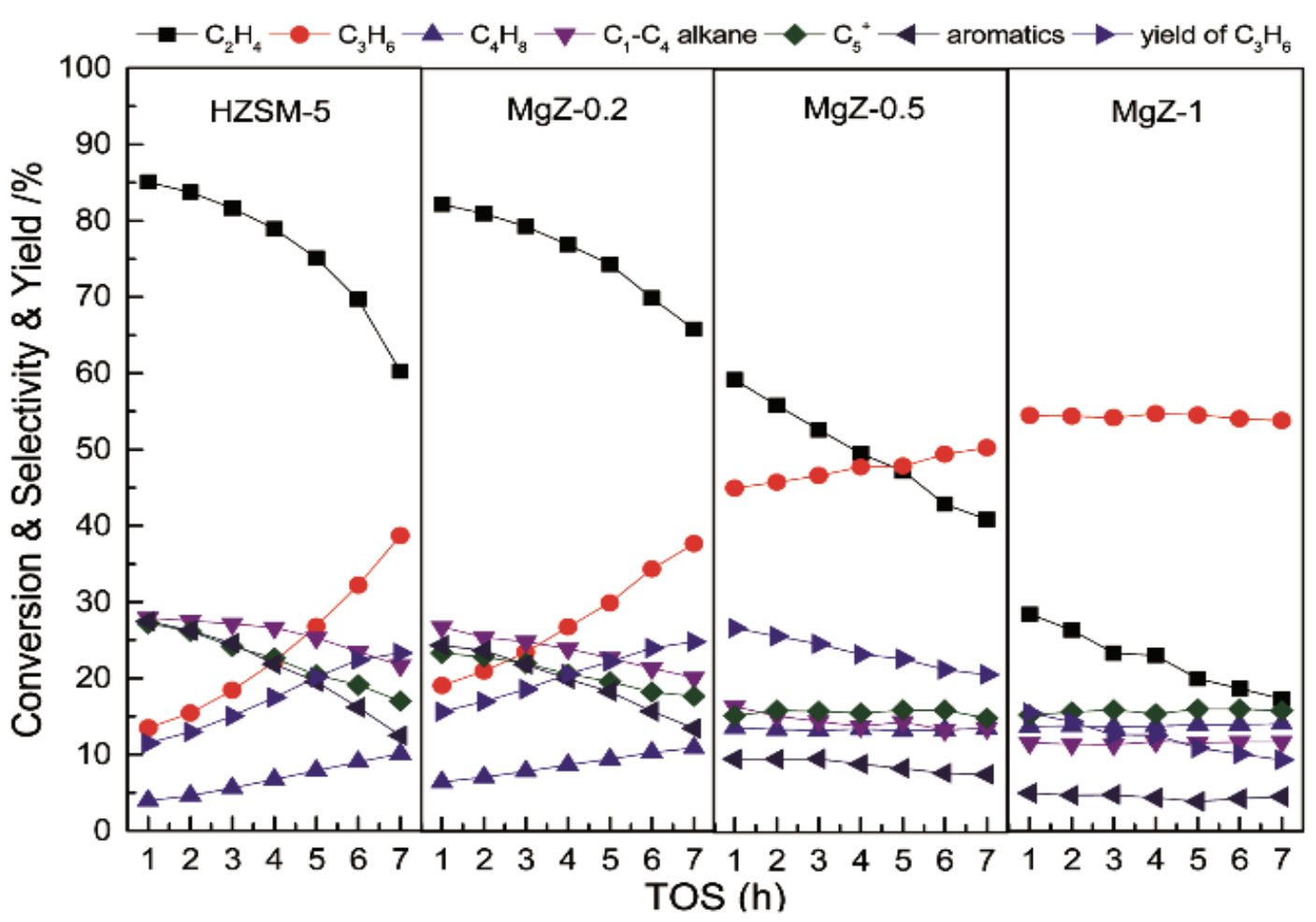

图 6 不同 Mg 改性 HZSM-5 分子篮对乙烯转化制 丙烯的催化性能

Fig. 6 Catalytic performances of HZSM-5 zeolites modified with different Mg contents for ETP reaction.

转化率下降不明显, 但是丙烯选择性也没有明显 提高, 所以初始丙烯产率较低, 但由于反应过程中 生成的积碳会覆盖一部分强酸位进而会提高丙烯 选择性, 所以丙烯产率逐渐增加; 而0.5\% Mg改性 样品初始丙烯产率远高于 HZSM-5, 这表明 Mg 含 量增加后覆盖了部分强酸性位进而提高了丙烯选 择性, 而随着反应的进行, 积碳产生使酸性位覆盖 导致乙烯转化率降低(由6 0 \% 降至 $45 \%$ ), 故丙烯产 率下降。 $1.0 \% \mathrm{Mg}$ 改性样品中 $\mathrm{Mg}$ 含量过高使得乙 烯转化率大大降低, 直接影响了丙烯的收率。上述 结果表明, 适量的 $\mathrm{Mg}$ 改性可以提高目的产物丙烯 的选择性, 但是过量 $\mathrm{Mg}$ 改性会导致反应活性下降 明显。

\section{6 反应后积碳样品的 TPO表征}

程序升温氧化(TPO)技术可以用来确认不同 类型的积碳物种。图7为经过ETP反应后不同积碳 样品的 TPO曲线。其中在 $450{ }^{\circ} \mathrm{C}$ 左右的低温烧碳峰 归属为高 $\mathrm{H} / \mathrm{C}$ 比的积碳, 在 $600-650^{\circ} \mathrm{C}$ 左右的高温 烧碳峰归属为低 $\mathrm{H} / \mathrm{C}$ 比的积碳。一般认为高温烧碳 峰是分子筱B酸位上形成的积碳 32,33 。随着 HZSM5 分子篎负载 $\mathrm{Mg}$ 的增加, 高温烧碳峰代表的积碳 量逐渐减少, 并且积碳氧化峰温由 $650{ }^{\circ} \mathrm{C}$ 向 $600{ }^{\circ} \mathrm{C}$ 移动, 同时在 $450^{\circ} \mathrm{C}$ 左右明显出现低温氧化峰。这
说明 Mg改性HZSM-5 后, 样品中贫氢积碳逐渐变 为富氢积碳, 同时也表明催化剂的强 $\mathrm{B}$ 酸含量减 少。与前面 $\mathrm{NH}_{3}-\mathrm{TPD}$ 和pyridine-IR 的表征结果一 致。

3.7 反应后积碳样品的 ${ }^{13}$ C CP/MAS NMR表征

图8给出了 Mg改性HZSM-5催化剂积碳样品 的 ${ }^{13} \mathrm{C} \mathrm{CP} / \mathrm{MAS}$ NMR谱图。可以看出在 $\delta=10-40$ 处 出现一个宽化的共振峰, 同时在 $\delta=24$ 和 $\delta=31$ 处

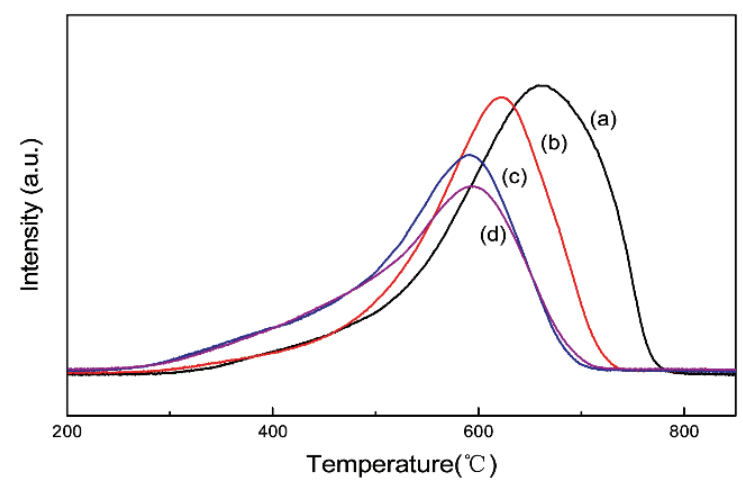

图 7 反应后不同积碳 Mg/HZSM-5 催化剂的 TPO 曲线

Fig. 7 TPO curves of coked Mg/HZSM-5 catalysts after reaction.

(a) HZSM-5, (b) MgZ-0.2, (c) MgZ-0.5, (d) MgZ-1. 


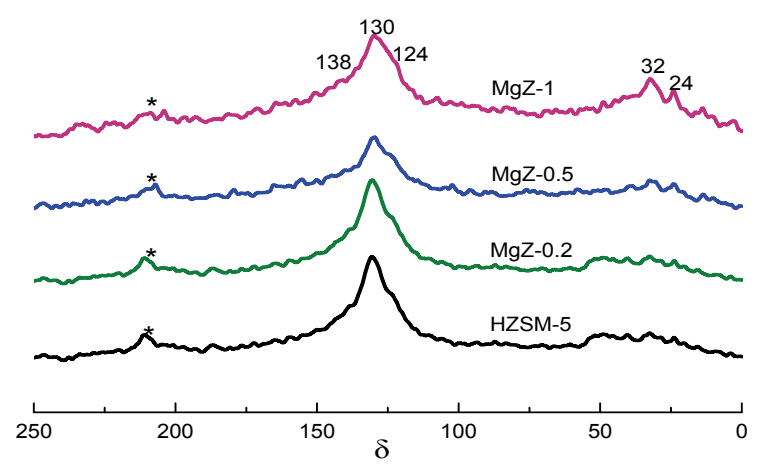

图 8 反应后不同积碳 Mg/HZSM-5 催化剂的 ${ }^{13} \mathrm{C}$ CP/MAS NMR 谱

Fig. $8{ }^{13} \mathrm{C} \mathrm{CP} / \mathrm{MAS}$ NMR spectra of coked Mg/HZSM-5 catalysts after reaction.

* denotes the spinning sidebands.

有相对较强的尖峰, 这是长链聚合物、芳烃或者烯 烃上含有 $\mathrm{CH}_{2}$ 基团的共振峰 ${ }^{3}$ 。表明长时间反应过 程中乙烯等烯烃分子发生了聚合反应生成了聚烯 烃类的积碳。此外, $\delta=124-138$ 左右处的信号归 属为芳烃的共振峰 34 。图 8 表明ETP反应过程中产 生的积碳主要是贫氢的稠芳烃类物种, 同时也有 少部分的富氢脂肪烃类积碳。这与上面 $\mathrm{TPO}$ 表征 结果一致。

由以上结果可知 HZSM-5 分子篎的酸性对 ETP 反应性能影响很大, 经过 Mg 改性后 HZSM5 分子篮的酸性发生了不同程度的变化, 这使 ETP 反应性能显著改善。由 $\mathrm{NH}_{3}$-TPD 和 pyridine-IR 表 征结果可以看出, $0.5 \% \mathrm{Mg}$ 改性后 HZSM-5 分子 簰的总酸量和强 B 酸数量明显减少, 而中强酸数 量有所增加。 ${ }^{29}$ Si MAS NMR 显示该催化剂虽然有 少量脱铝, 但是 XRD 和 BET 比表面积测定表明 其骨架结晶度保持良好, 如此的结构和酸性质保 障其在 ETP 反应中提升丙烯选择性达到 $45 \%$ 以 上, 并且乙烯的转化率也能维持在 50\%左右。1.0\% 过量 Mg 改性 HZSM-5 使得强 B 酸和中强酸数量 均下降, 并且其骨架结晶度也有下降, 从而导致了 乙烯转化率的明显下降。这说明 HZSM-5 中保持 合适的强 $\mathrm{B}$ 酸和中强酸数量对于获得高丙烯收率 至关重要, 因为这有利于保持较高的乙烯转化率, 使低碳烯烃发生氢转移、缩聚并芳构化成积碳的 几率降低, 从而使得目的产物丙烯的选择性增加。 Dai 等人 12 在八元环的小孔 SSZ-13 分子篮上进行 ETP 反应也发现在分子篮脱铝后 B 酸量的降低可 以减少烷烃、芳烃等副产物的选择性。这对应于 TPO 表征结果, Mg 改性后生成的稠芳烃积碳明显 减少。但是强 $\mathrm{B}$ 酸量的过量减少也会伴随着反应
活性的明显降低, 因此在增加中强酸量的同时也 要保持一定的强 B 酸位, 从而使催化剂的 ETP 反 应性能达到最佳。

\section{4 结论}

Mg改性可以有效调变HZSM-5 分子篮催化剂 的结构和酸性, 改性后的HZSM-5 分子篮催化剂在 乙烯制丙烯反应中表现出优良的催化性能。在 $550^{\circ} \mathrm{C}$ 、乙烯体积空速(GHSV)为 $3000 \mathrm{~h}^{-1}$ 的最佳条 件下, $0.5 \%$ 适量镁改性HZSM- 5 导致乙烯转化率有 所下降, 但丙烯选择性增加到 $45 \%$ 以上, 而芳烃的 选择性降到 $8 \%$ 以下。 $\mathrm{NH}_{3}$-TPD和pyridine-IR 表征 结果显示适量 Mg改性后HZSM-5分子篎的总酸 量和强 $\mathrm{B}$ 酸数量明显减少, 而中强酸数量有所增 加。0.5\% Mg/HZSM-5 上具有适当含量的强B酸和 中强酸中心, 从而提高了ETP反应中丙烯的选择 性, 降低了低碳烷烃和芳烃的选择性。但是过量的 镁改性使分子篎的总酸量明显减少, 导致催化剂 的活性显著下降。

\section{References}

(1) Shi, L.; Wang, D. Q.; Song, W.; Shao, D.; Zhang, W. P.; Lu, A. H. ChemCatChem 2017, 9 (10), 1788.

doi: $10.1002 /$ cctc. 201700004

(2) Sattler, J. J. H. B.; Ruiz-Martinez, J.; Santillan-Jimenez, E.;

Weckhuysen, B. M. Chem. Rev. 2014, 114 (20), 10613. doi: $10.1021 / \mathrm{cr} 5002436$

(3) Lu, H. Q.; Shi, L.; He, C.; Weng, W. Z.; Huang, C. J.; Wan, H. L. Acta Phys. -Chim. Sin. 2012, 28 (11), 2697. [鲁怀乾, 石䂞, 何冲, 翁维正, 黄传敬, 万惠霖. 物理化学学报, 2012, 28 (11), 2697.] doi: 10.3866/PKU.WHXB201207091

(4) Mol, J. C. J. Mol. Catal. A 2004, 213 (1), 39. doi: 10.1016/j.molcata.2003.10.049

(5) Li, X. J.; Zhang, W. P.; Liu, S. L.; Xu, L. Y.; Han, X. W.; Bao, X. H. J. Catal. 2007, 250 (1), 55. doi: 10.1016/j.jcat.2007.05.019

(6) Li, X.; Zhang, W. P.; Li, X. J.; Liu, S. L. Prog. Chem. 2008, 20 $(7 / 8), 1021$. [李新, 张维萍, 李秀杰, 刘盛林, 化学进展, 2008, $20(7 / 8), 1021$.

(7) Liu, Z. F. Acta Phys. -Chim. Sin. 2016, 32 (4), 803. [刘忠范. 物理 化学学报, 2016, 32 (4), 803.] doi: 10.3866/PKU.WHXB2016032801

(8) Xu, S. T.; Zheng, A. M.; Wei, Y. X.; Chen, J. R.; Li, J. Z.; Chu, Y. Y.; Zhang, M. Z.; Wang, Q. Y.; Zhou, Y.; Wang, J. B. Angew. Chem. Int. Ed. 2013, 52 (44), 11564. doi: 10.1002/anie.201303586

(9) Wu, X. Q. ; Xu, S. T.; Zhang, W. N.; Huang, J. D.; Li, J. Z.; Yu, B. 
W.; Wei Y. X.; Liu, Z. M. Angew. Chem. Int. Ed. 2017, 56 (31), 9039. doi: 10.1002/anie.201703902

(10) Tian, P.; Wei, Y.; Ye, M.; Liu, Z. ACS Catal. 2015, 5 (3), 1922. doi: $10.1021 /$ acscatal.5b00007

(11) Hu, S.; Zhang, Q.; Gong, Y. J.; Zhang, Y.; Wu, Z. J.; Dou, T. Acta Phys. -Chim. Sin. 2016, 32 (7), 1785. [胡思, 张卿, 巩雁军, 张瑛, 吴志杰, 窦涛. 物理化学学报. 2016, 32 (7), 1785.] doi: 10.3866/PKU.WHXB201604152

(12) Dai, W. L.; Sun, X. M.; Tang, B.; Wu, G. J.; Li, L. D.; Guan, N. J.; Hunger, M. J. Catal. 2014, 314, 10. doi: 10.1016/j.jcat.2014.03.006

(13) Oikawa, H.; Shibata, Y.; Inazu, K.; Iwase, Y.; Murai, K.; Hyodo, S.; Kobayashi, G.; Baba, T. Appl. Catal. A: Gen. 2006, 312, 181. doi: 10.1016/j.apcata.2006.06.045

(14) Iwase, Y.; Motokura, K.; Koyama, T. -R.; Miyaji, A.; Baba, T. Phys. Chem. Chem. Phys. 2009, 11 (40), 9268. doi: 10.1039/b911659a

(15) Koyama, T. -R.; Hayashi, Y.; Horie, H.; Kawauchi, S.; Matsumoto, A.; Iwase, Y.; Sakamoto, Y.; Miyaji, A.; Motokura, K.; Baba, T. Phys. Chem. Chem. Phys. 2010, 12 (11), 2541. doi: $10.1039 / \mathrm{b} 921927 \mathrm{~g}$

(16) Lin, B. M.; Zhang, Q. H.; Wang, Y. Ind. Eng. Chem. Res. 2009, 48 (24), 10788. doi: 10.1021/ie901227p

(17) Follmanna, S.; Ernst, S. New J. Chem. 2016, 40 (5), 4414. doi: $10.1039 / \mathrm{C} 5 \mathrm{NJ} 03668 \mathrm{~B}$

(18) Ikeda, K.; Kawamura, Y.; Yamamoto, T.; Iwamoto, M. Catal. Commun. 2008, 9 (1), 106. doi: 10.1016/j.catcom.2007.05.032

(19) Alvarado Perea, L.; Wolff, T.; Veit, P.; Hilfert, L.; Edelmann, F. T.; Hamel, C.; Seidel-Morgenstern, A. J. Catal. 2013, 305, 154. doi: 10.1016/j.jcat.2013.05.007

(20) Bleken, F. L.; Chavan, S.; Olsbye, U.; Boltz, M.; Ocampo, F.; Louis, B. Appl. Catal. A: Gen. 2012, 447, 178. doi: 10.1016/j.apcata.2012.09.025

(21) Mao, D. S.; Guo, Q. S.; Meng, T; Acta Phys.-Chim. Sin. 2010, 26 (8), 2242. [毛东森, 郭强胜, 孟涛. 物理化学学报, 2010, 26 (8),
2242.] doi:10.3866/PKU.WHXB20100814

(22) Hu, S.; Zhang, Q.; Xia, Z.; Gong, Y. J.; Xu, J.; Deng, F.; Dou, T. Acta Phys. -Chim. Sin. 2015, 31 (7), 1374. [胡思, 张卿, 夏至, 巩 雁军, 徐君, 邓风, 窦涛. 物理化学学报, 2015, 31 (7), 1374.] doi: 10.3866/PKU.WHXB201504302

(23) Lehmann, T.; Wolff, T.; Zahn, V. M.; Veit, P.; Hamel, C.; SeidelMorgenstern, A. Catal. Commun. 2011, 12 (5), 368. doi: 10.1016/j.catcom.2010.10.018

(24) Iwase, Y.; Sakamoto, Y.; Shiga, A.; Miyaji, A.; Motokura, K.; Koyama, T. -R.; Baba, T. J. Phys. Chem. C 2012, 116 (8), 5182. doi: $10.1021 /$ jp212549j

(25) Tanaka, M.; Itadani, A.; Kuroda, Y.; Iwamoto, M. J. Phys. Chem. C 2012, 116 (9), 5664. doi: 10.1021/jp2103066

(26) Zhang, W. P.; Bao, X. H.; Guo, X. W.; Wang, X. S. Catal. Lett. 1999, 60 (1/2), 89. doi: A:1019061714047

(27) Zhang, W. P.; Xu, S. T.; Han, X. W.; Bao, X. H. Chem. Soc. Rev. 2012, 41, 192. doi: 10.1039/c1 cs15009j

(28) Mao, D. S.; Yang, W. M.; Xia, J. C.; Zhang, B.; Song, Q. Y.; Chen, Q. L. J. Catal. 2005, 230 (1), 140. doi: 10.1016/j.jcat.2004.12.007

(29) Bai, J.; Liu, S. L.; Xie, S. J.; Xu, L. Y.; Lin, L. W. Chin. J. Catal. 2004, 25 (1), 70. [白杰, 刘盛林, 谢素娟, 徐龙伢, 林励吾. 催 化学报, 2004, 25 (1), 70.]

(30) Topsoe, N. Y.; Pedersen, K.; Derouane, E. G. J. Catal. 1981, 70 (1), 41. doi: 10.1016/0021-9517(81)90315-8

(31) Emeis, C. A. J. Catal. 1993, 141 (2), 347. doi: $10.1006 /$ jcat.1993.1145

(32) Ma, D.; Shu, Y. Y.; Cheng, M. J.; Xu, Y. D.; Bao, X. H. J. Catal. 2000, 194 (1), 105. doi: 10.1006/jcat.2000.2908

(33) Li, X. J.; Zhang, W. P.; Li, X.; Liu, S. L.; Huang, H. J.; Han, X. W.; Xu, L. Y.; Bao, X. H. J. Phys. Chem. C 2009, 113 (19), 8228. doi: $10.1021 /$ jp901103e

(34) Bonardet, J. L.; Barrage, M. C.; Fraissard, J. J. Mol. Catal. A: Chem. 1995, 96 (2), 123. doi: 10.1016/1381-1169(94)00030-1 